\title{
FICCIÓN, HISTORIA Y VERDAD: ESTUDIO DE LA INTERTEXTUALIDAD EN LA SCOMPARSA DI MAJORANA DE LEONARDO SCIASCIA
}

\author{
Javier Serrano Puche \\ Universidad de Navarra \\ jserrano@unav.es \\ RESUMEN / ABSTRACT
}

El artículo examina la variedad y abundancia de referencias intertextuales presentes en $L a$ scomparsa di Majorana (1975) de Leonardo Sciascia, un relato de investigación (raccontoinchiesta) en el que el escritor siciliano reconstruye un hecho histórico-la desaparición en 1938 del científico Ettore Majorana-con la ayuda no solo de fuentes historiográficas y documentales, sino también de motivos y elementos ficcionales, desde la convicción de que la literatura ayuda a iluminar la realidad y es el espacio donde la verdad halla su mayor cumplimiento.

Palabras clave: Leonardo Sciascia, intertextualidad, verdad literaria, investigación histórica, Ettore Majorana.

The paper examines the variety and abundance of intertextual references present in 'The Mystery of Majorana' (1975), a narrative research (racconto-inchiesta) in which the Sicilian writer Leonardo Sciascia reconstructs a historical event: the disappearance in 1938 of the scientist Ettore Majorana. Sciascia does so not only with the help of historical sources and documents, but also literary motifs and devices, because he is convinced that literature, understood as a space where truth takes place, helps to illuminate reality.

KEY WORDS: Leonardo Sciascia, intertextuality, literary truth, historical research, Ettore Majorana. 


\section{EL RACCONTO-INCHIESTA COMO GÉNERO LITERARIO}

La obra del escritor italiano Leonardo Sciascia (1921-1989) es abundante y compleja, reflejo de una no menos rica personalidad y maduración intelectual. Más de treinta títulos entre poemarios, obras de teatro, novelas, libros de relatos, ensayos, estudios historiográficos, diarios y antologías de sus columnas periodísticas componen la producción de un escritor fundamental en la literatura europea de la segunda mitad del siglo XX. Si bien la escritura sciasciana no suele acomodarse dentro de los límites de un género concreto -y en una misma obra puede asumir alternativamente la forma ensayística, narrativa o de indagación- es posible señalar, junto con la ficción ${ }^{1}$, un subgénero preeminente en la creación literaria del autor siciliano: el relato de investigación o racconto-inchiesta.

Según declaró Sciascia (Nigro 107), el modelo de sus racconti-inchiesta era la Storia della colonna infame ${ }^{2}$, con la que Alessandro Manzoni inauguró el género en 1842. De esta obra, Sciascia aprendió a tomar como punto de partida algún hecho real, histórico, sobre el que se documentaba (a través de la prensa de la época, de actas judiciales, informes de la policía, diarios y testimonios de los protagonistas...) para luego reconstruirlo por medio de la narración y la inclusión en el relato de algunos de dichos documentos. Por su enfoque centrado sobre los acontecimientos menores y significativos y, sobre todo, por su dimensión narrativa, este procedimiento lo aproxima a la microhistoria, corriente historiográfica que alcanzó su apogeo en la década de 1970, simultáneamente a la publicación de los racconti-inchiesta sciascianos,

${ }^{1}$ Entre las obras de ficción de Sciascia tiene cabida tanto la narración breve (conjunto de relatos como Gli zii di Sicilia (1958) o Il mare colore del vino (1973)) como la novelística, que a su vez comprende obras de diferentes subgéneros: novela histórica (Il consiglio d'Egitto, escrita en 1963), de ambiente judicial (Porte aperte (1987), la reescritura de un clásico literario (Candido ovvero un sogno fatto in Sicilia, de 1977) o de una fábula del folclore siciliano como Cola Pesce (1975). Mención aparte merece el subgénero policíaco, por las seis novelas que escribió Sciascia, que se encuentran entre las más destacadas de su producción literaria: Il giorno della civetta (1961), A ciascuno il suo (1966), Il contesto (1971), Todo modo (1974), Il cavaliere e la morte (1988) y Una storia semplice (1989).

${ }^{2}$ La Storia della colonna infame (Manzoni) es la relación del proceso judicial que se desarrolló en Milán con ocasión de la peste de 1630 y en el que fueron injustamente condenados a muerte un grupo de hombres, acusados de ser los propagadores de la plaga. El tema ya aparece en I promessi sposi (caps. XXXI-XXXVII) y en un principio Manzoni pensó que fuera un apéndice de la novela, aunque finalmente apareció publicada de modo independiente. 
y que se desmarcaba de la historiografía convencional, de la que demostró el autor siciliano una desconfianza creciente (Sciascia, La Sicilia 82).

Muchos de los racconti-inchiesta escritos por Sciascia relatan algún proceso judicial o investigación de la policía, ya sea durante la vigencia de la Inquisición (como Morte dell' inquisitore (1964), La sentenza memorabile (1982), La strega e il capitano (1986) y alguna de las Cronachette (1985)) o bien de épocas más recientes a las que la narración de aquel acontecimiento ayuda a iluminar. Es el caso de I pugnalatori (1976) respecto de la Unificación Italiana o del relato $1912+1$, escrito en 1986 y que recrea un caso judicial que transcurre en la Italia de comienzos del siglo XX. También el régimen fascista - Atti relativi alla morte di Raymond Roussel (1972), La scomparsa di Majorana (1975), Il teatro della memoria (1981) - o el gobierno de la Democracia Cristiana (por medio de L'affaire Moro (1978) o Dalle parte degli infedeli (1979)) son el escenario donde se desarrollan los hechos de algunos relatos de investigación.

La lectura perspicaz que realiza el narrador de los documentos disponibles sobre cada uno de los casos narrados desvela con frecuencia errores y omisiones en la investigación, contradicciones y falta de rigor en el desarrollo de procesos que a menudo concluyen con la condena injusta de personas inocentes o con la absolución de los culpables. Al mismo tiempo, como elemento de interpretación e instrumento que ayude a paliar las lagunas y la escasez de documentos, Sciascia se sirve continuamente de la ficción. Por medio de la apelación a fragmentos de otras obras, a circunstancias biográficas de escritores, a personajes o motivos literarios, intenta perfilar los rasgos de carácter de las personas que protagonizan el relato o entender lo que realmente ocurrió y no encuentra verdadera explicación en los textos que hasta entonces daban cuenta del suceso en cuestión.

La comprensión de la realidad se lleva a cabo, en definitiva, desde una perspectiva literaria, que tiene en las operaciones de lectura, escritura y reescritura sus instrumentos heurísticos más penetrantes. Con la convicción profesa del autor de que la única forma posible de verdad es la artística (Sciascia, La Sicilia 87), la realidad-sometida a la contingencia y maleable por las mentiras que a menudo provienen de las instituciones de poder-es la que ha de adecuarse al patrón literario y nunca a la inversa.

Leonardo Sciascia alcanzó dicha convicción sobre la dimensión veritativa del arte tras escribir La scomparsa di Majorana, un racconto-inchiesta que apareció por entregas en las páginas del diario La Stampa entre el 31 de 
agosto y el 7 de septiembre de 1975, y que en octubre publicaría la editorial Einaudi junto con diez notas añadidas por el autor.

La obra gira en torno a la misteriosa desaparición en marzo de 1938 del joven físico Ettore Majorana, que había comunicado a un amigo su intención de suicidarse y cuyo rastro se perdió poco después en una travesía en barco entre Nápoles y Palermo. Majorana trabajaba en el grupo de científicos que bajo la dirección de Enrico Fermi descubrió la escisión del átomo, hallazgo que precedió a la fabricación de la bomba atómica. La hipótesis que defiende Sciascia en su racconto-inchiesta es que, dado su carácter de genio como atestiguan quienes le conocieron, Majorana pudo intuir con bastante antelación que el fruto de sus investigaciones podría ser utilizado como un arma mortífera y, rechazando tal posibilidad por inmoral, decidió desaparecer, pero haciendo creer que se había suicidado. Esta hipótesis se sustenta sobre una sólida labor de investigación en la que Sciascia maneja todas las fuentes primarias disponibles (epistolario del protagonista de los hechos, documentos policiales sobre la investigación del caso, testimonios de sus compañeros de laboratorio, ensayos científicos, crónicas periodísticas, etc.), enmarcando además los hechos en su contexto político-social, cultural y científico. Según el escritor -y en esto difiere de otros estudiosos de la vida de Majorana como Russo (1997), Recami (2000), o Magueijo (2009)- quizá el científico nacido en Catania se refugió en un convento del sur de Italia, aunque su relato de investigación concluye a sus puertas y sin que pueda demostrarlo fehacientemente.

No obstante, el narrador-Sciascia no se siente decepcionado, sino todo lo contrario puesto que, más allá de la racional certeza del paradero del físico siciliano, confiesa haber vivido una experiencia de revelación plenamente literaria que no está supeditada a su constatación fáctica en la realidad. Dicha experiencia es el culmen de las continuas referencias a fragmentos, motivos y personajes literarios a los que acude la instancia narrativa de $L a$ scomparsa di Majorana para comprender mejor todo lo concerniente a la vida y desaparición del científico.

En las páginas siguientes señalaremos las formas de intertextualidad que aparecen en este racconto-inchiesta y que constituyen una tipología muy variada de recursos mediante los cuales la instancia narrativa acude a otras voces, literarias o artísticas, en su intento de comprensión de la realidad. No consideraremos aquí, pues excede las posibilidades de este artículo, el modo en que es empleada en La scomparsa di Majorana toda aquella documentación oficial, científica, epistolar o testimonial disponible acerca 
de la vida y desaparición de Ettore Majorana, sino que nos centraremos en la intertextualidad funcional poética presente en el relato. Para ello, como ha señalado Millán Alba, prestaremos atención a "la presencia en un texto, de otro (u otros) ajenos al primero, pero que, sin embargo, resultan ser funcionales respecto de aquél; esto es, funcionan estructuralmente en su configuración estrictamente singular, constituyendo una de las hiladas de la urdimbre textual" (11). En este sentido, para que un intertexto sea funcional, no se agote en su categoría referencial de fuente y acceda a la categoría de función intertextual, según Del Prado Biezma son necesarias dos cualidades:

La primera, intrínseca, reside en la capacidad germinativa del intertexto, en su capacidad para prender y fecundar el nuevo espacio textual en el que se injerta: esta capacidad está ligada, sin lugar a dudas, al potencial simbólico que arrastra. La segunda, extrínseca, recae de nuevo sobre el espacio de la recepción: el lector debe ser capaz de captar la intertextualidad, como ha sido capaz de captar la referencialidad primaria - de no ser así, el texto cobrará una función que podemos llamar exótica. Esta captación extrae al lector de la clausura del texto único y cerrado, y lo proyecta de manera problemática, interrogativa y dialéctica, en el ámbito de la architextualidad (409).

En el caso de La scomparsa di Majorana examinaremos, pues, cómo el relato se asienta sobre un tejido polifónico con resonancias directas e indirectas de otras obras, personajes y autores que lo habilitan como espacio donde la verdad acontece.

\section{PERSONAJES DE FICCIÓN Y CITACIÓN DE OBRAS LITERA- RIAS}

En La scomparsa di Majorana, Sciascia confiesa que su motivación para escribir el relato de investigación es el deseo de comprender el drama moral sufrido por el científico siciliano que le llevó a tomar la decisión de suicidiarse $\mathrm{o}$, como sostiene aquel, a renunciar, trágicamente y por motivos de conciencia, a su carrera investigadora en la física nuclear. Comprender el drama para luego narrarlo al lector presupone, en primer lugar, conocer, a través de los hechos y datos relativos a su vida, la personalidad del físico. 
Como esos elementos son escasos e insuficientes para demostrar qué pudo inducir a Majorana a desaparecer, Sciascia intenta adentrarse en la psicología del joven para, en último término, poder "encontrarlo" si no ya físicamente, sí en su verdad íntima y revelarla epifánicamente en su relato.

Es consciente de la dificultad de su misión; más aún, piensa que solo un investigador como Charles Dupin, el personaje de ficción creado por E. A. Poe, habría aceptado un reto de dicha envergadura (Sciascia, La scomparsa 217). En consecuencia, hace propio el método del clásico detective, como volverá a suceder en 1978 en el relato de investigación L'affaire Moro $^{3}$, donde hace explícito aquello que según el personaje del escritor norteamericano es el fundamento de toda investigación: la capacidad de identificarse, de sumirse en el otro (Sciascia, L'affaire Moro 489).

No solo en el orden metadiscursivo se recurre a la comparación con personajes o motivos literarios para intentar ilustrar el drama de Ettore Majorana. Uno de los dos epígrafes que encabezan el racconto-inchiesta, tomado de la Nota biografica di Ettore Majorana escrita por el científico Edoardo Amaldi, afirma que William Shakespeare y Luigi Pirandello eran los escritores favoritos de Majorana (Sciascia, La scomparsa 207). Desde la perspectiva asumida por la instancia narrativa es coherente, pues, que sea entre las obras de aquellos donde quepa encontrar los personajes que mejor se le asemejan o que incluso hayan podido inspirar al científico en sus planes de desaparición.

3 El 16 de marzo de 1978 las Brigadas Rojas secuestraron a Aldo Moro, presidente del Consejo nacional de la Democracia Cristiana (DC), cuando se dirigía a la presentación del nuevo gobierno de Giulio Andreotti, el primero que contaba con el apoyo del Partido Comunista Italiano (PCI). Después de 55 días de secuestro, el 9 de mayo apareció el cadáver de Moro dentro del maletero de un coche que, como concreción simbólica del compromesso storico, del que aquel era principal valedor, fue abandonado por los secuestradores en una calle situada a medio camino entre las sedes romanas de la DC y el PCI. Este acontecimiento inspiró a Sciascia escribir L'affaire Moro, un racconto-inchiesta en el que el escritor analizaba con rigor filológico las cartas que el líder democristiano había enviado durante su secuestro, los comunicados de las Brigadas Rojas y las reacciones de la clase política ante el secuestro, llegando a la conclusión de que los compañeros de partido de Moro no hicieron todo lo posible para salvarle, pues su captura en realidad favorecía el clima de tranquilidad y concordia en que se había aprobado el gobierno de Andreotti y en el que finalmente contarían con la colaboración de los comunistas. Para un estudio pormenorizado de la obra y de la fuerte polémica generada en el ámbito cultural y político italiano, cfr. Vecellio (2002). 
Esto es particularmente claro respecto del dramaturgo siciliano y, más concretamente, con los protagonistas de sus novelas Il fu Mattia Pascal y Uno, nessuno e centomila. Una posible inspiración que también fue sugerida por los medios de comunicación que informaron de aquel suceso y que la instancia narrativa reconduce hasta proponer el personaje más adecuado para la comparación:

Se si ha l'accortezza o la vocazione di non tornare a intricarsi con "gli altri", di guardare alla loro vita e ai loro sentimenti con l'occhio di un entomologo; accortezza o vocazione di cui mancò del tutto Mattia Pascal ed ebbe invece, più di vent'anni dopo, Vitangelo Moscarda: e ricordiamo questi due personaggi pirandelliani anche per il fatto che a livello giornalistico e televisivo è stata data per certa un'affezione, come a modello, di Ettore Majorana a Mattia Pascal; mentre più si confaceva alle sue aspirazioni il protagonista di Uno, nessuno e centomila (Sciascia, La scomparsa 261) ${ }^{4}$.

Por tanto, la situación que vivió el físico se asemejaría, más que a la de Mattia Pascal, a la de Vitangelo Moscarda, puesto que Majorana comparte con éste el deseo de estar solo ${ }^{5}$; deseo que provoca que sienta como una trampa las obligaciones de la vida cotidiana (Sciascia, La scomparsa 251).

${ }^{4}$ Ofrecemos, en nota a pie, una traducción española de los fragmentos citados en italiano a lo largo de este artículo: "Si se tiene la cautela o la vocación de no volver a trabar relación con los demás, de observar su vida y sus sentimientos con ojos de entomólogo; cautela o vocación de las cuales carecía por completo Mattia Pascal y que tuvo, en cambio, veinte años después, Vitangelo Moscarda; y traemos a colación estos dos personajes de Pirandello, también por el hecho de que a nivel periodístico y televisivo se dio por cierta la afición de Ettore Majorana a tomar como modelo a Mattia Pascal, cuando, en realidad, el protagonista de Uno, ninguno $y$ cien mil se ajustaba mucho más a sus aspiraciones".

${ }^{5}$ Así formula su deseo Vitangelo Moscarda ante el lector: "Io volevo esser solo in un modo affatto insolito, nuovo. Tutt'al contrario di quel che pensate voi: cioè senza me e appunto con un estraneo attorno. Vi sembra già questo un primo segno di pazzia? Forse perché non riflettete bene. Poteva già essere in me la pazzia, non nego; ma vi prego di credere che l'unico modo d'esser soli veramente è questo che vi dico" (Pirandello 1292). Es decir: "Quería estar solo de un modo del todo insólito, nuevo. Todo lo contrario de lo que pensáis, es decir, sin mí, más aún, con un extraño alrededor. ¿Os parece ya esto un primer síntoma de locura? Quizá porque no reflexionáis bien. Podía estar ya en mí la locura, no lo niego, pero os ruego que creáis que el único modo de estar verdaderamente solo es este que os digo". 
También en los cuentos y novelas de Vitaliano Brancati ${ }^{6}$ aparecen personajes que, por vía de la comparación, pueden ayudar a explicar la psicología de Majorana, como señala el narrador en el capítulo VII: "E pensiamo, si capisce, a quei personaggi marginali, come Ermenegildo Fasanaro nel Bell'Antonio, che sentono lo spavento di quella specie di "fissione umana", di scatenarsi dell'energia del male nell'uomo, che avviene (1939-1945) sotto i loro occhi; e specialmente pensiamo al protagonista del racconto La cimice" (Sciascia, La scomparsa 248-249)7.

De este modo, los límites de La scomparsa di Majorana se ensanchan y abarcan más allá del propio texto, su lectura está abierta al reclamo de muchas otras obras, que conviene conocer o a las que hay que acudir para comprender en toda su riqueza el relato de investigación sciasciano. Para un conocimiento más profundo del carácter de Majorana y de sus miedos, por tanto, el lector habría de conocer los rasgos de aquel personaje de la comedia Il bell'Antonio y del protagonista del cuento "La cimice".

Igualmente, ha de remitirse al Canto XXVI del "Infierno" de la Divina Commedia para leer la escena de la muerte del Ulises recreado por Dante y entender así el mito que, según el narrador del racconto-inchiesta, encarna el científico siciliano con su supuesta muerte por ahogamiento (Sciascia, $L a$ scomparsa 261). Así pues, sea real o simulada, con su desaparición en las aguas marinas, Majorana estaría repitiendo la suerte del Ulises dantesco, que murió después de haber salido de Ítaca en busca de nuevas aventuras (Alighieri 295).

La scomparsa di Majorana no se enriquece intertextualmente solo por medio de las semejanzas que pueden establecerse entre la persona sobre la que gira todo el relato y determinados personajes de ficción, como hemos señalado hasta ahora. Junto a esto, existe otro crecimiento y densificación de

${ }^{6}$ Nació en Pachino (Siracusa) en 1907 y murió en 1954 en Turín. Escribó obras de teatro, cuentos y novelas, en un primer momento marcadas por la admiración al fascismo (Fedor 1928; Piave 1932), que más tarde repudiaría y sobre el que ironizaría, como en Raffaele (1946), de la que La scomparsa di Majorana reproduce dos escenas. Son notables también sus comedias de tono satírico y erótico como Don Giovanni in Sicilia (1942) e Il bell'Antonio (1949).

7 "Y pensamos, se entiende, en aquellos personajes marginales, como Hermenegildo Fasanaro en Bell'Antonio, que sienten el terror de aquella especie de 'fisión humana' del desencadenarse del mal del hombre que se desarrolla (1939-1945) ante sus ojos; y pensamos especialmente en el protagonista del cuento La chinche". 
la narración con la inclusión explícita o implícita de versos, frases o diálogos de otras obras literarias.

En virtud de lo anterior, no solo los protagonistas de los libros de Brancati, como ya vimos, pueden ayudar a definir el miedo de Majorana. También la poesía y, en concreto, "versi di Eliot o di Montale potrebbero aiutarci a definire il suo "spavento"” (Sciascia, La scomparsa 248) ${ }^{8}$. A primera vista parece una invocación como las que examinábamos arriba, que remite al exterior del relato, a unos versos de T. S. Eliot o de Eugenio Montale. De la familiaridad del lector con las obras de estos poetas dependerá el valor y efecto de la apelación, pues ésta queda condicionada a que el lector identifique unas palabras de aquellos como apropiadas para los avatares vitales del físico9. En el caso de Eliot, sin embargo, esa tarea de identificación queda resuelta, aunque no explícitamente, en el propio relato, cuando el narrador indica en el décimo capítulo: "Doveva essergli di angoscioso peso (...) intravedere quel peso di morte che sentiva di portare oggettivarsi nella particolare ricerca e scoperta di un segreto della natura: depositarsi, crescere, diffondersi nella vita umana come polvere mortale. In una manciata di polvere ti mostrerò lo spavento, dice il poeta. E questo spavento crediamo abbia visto Majorana in una manciata di atomi" (Sciascia, La scomparsa 263) ${ }^{10}$.

Sin citar el nombre de Eliot, Sciascia reproduce un verso del primer capítulo de La tierra baldía, "The burial of the dead", en el que el poeta exclama: "And I will show you something different from either / Your shadow at morning striding behind you / Or your shadow at evening rising to meet you / I will show you fear in a handful of dust" (Eliot 92).

8 "Versos de Eliot o de Montale podrían ayudarnos a definir su 'terror"”.

9 A este respecto, parecen idóneos los siguientes versos de Montale que-conjugados en singular- bien podría haber pronunciado Majorana ante Enrico Fermi y demás compañeros de investigación: "Non domandarci la formula che mondi possa aprirti, / sì qualche storta sillaba e secca come un ramo. / Codesto solo oggi possiamo dirti, / ciò che non siamo, ciò che non vogliamo". ("No nos pidas la fórmula que mundos pueda abrirte / sí alguna torcida sílaba, seca como una rama / Esto sólo podemos hoy decirte: lo que no somos, lo que no queremos"). Es la tercera y última estrofa del poema inicial de Ossi di sepia: "Non chiederci la parola che squadri da ogni lato..." (Montale 29).

10 "Debía serle un peso angustiante (...) [que] ese peso de muerte que sentía llevar puesto se materializaba en la particular investigación y descubrimiento de un secreto de la naturaleza, depositándose, creciendo, difundiéndose en la vida humana como polvo mortal. 'En un puñado de polvo te enseñaré el horror', dice el poeta. Y ese horror creemos que lo había visto Majorana en un puñado de átomos". 
Con la paráfrasis posterior (en la que el polvo es sustituido por los átomos) y valiéndose de la fuerza de la imagen eliotiana, el narrador del raccontoinchiesta simboliza el drama de Ettore Majorana: en un puñado de átomos él ha visto el horror que solo verán los demás cuando caigan las bombas sobre Hiroshima y Nagasaki; y frente ese horror no ha encontrado otra salida que su desaparición.

Más allá de este verso, es posible detectar más elementos comunes entre La scomparsa di Majorana y La tierra baldía, pues en ambas, donde la intertextualidad es abundante, hay referencias a la Divina Commedia de Dante y a La tempestad de Shakespeare. También en el poema del escritor británico, como indica el título del cuarto capítulo, hay una "Death by water" como la que quizá padeciera el físico de Catania (Eliot 122).

No obstante, el de Eliot no es el único ejemplo de cómo un verso se integra en el relato de La scomparsa di Majorana con la finalidad de confiar a las capacidades expresivas y gnoseológicas de la palabra poética aquello que la instancia narrativa no puede decir por sí sola en toda su plenitud. En el último capítulo -después de exponer el rumor según el cual un pasajero del avión B-29 pudo haberse refugiado en el mismo convento en el que quizá también estuvo Majorana- el narrador cita a Alberto Savinio, que estaba convencido de que las ruinas de Troya eran verdaderamente las que descubrió el arqueólogo alemán Heinrich Schliemann y no otras, por el hecho de que en la Primera Guerra Mundial aquellas fueron bombardeadas por un barco inglés bautizado con el nombre homérico de Agamennon. De modo análogo, la instancia narrativa cree que la confluencia de aquellos rumores dispares en un mismo lugar ha de tener necesariamente un sentido:

Se l'ira non ancora sopita di Agamennone non li avesse animati, perché mai quei cannoni avrebbero sparato su delle rovine in una landa? I nomi, non che un destino, sono le cose stesse. Assurdo e mistero in tutto, Giacinta: dice il poeta José Moreno Villa. In tuttò è invece "razionale" mistero di essenze e rispondenze, continua e fitta trama -da un punto all'altro, da una cosa all'altra, da un uomo all'altro- di significati: appena visibili, appena dicibili (Sciascia, $L a$ scomparsa 269 $)^{11}$.

11 "Si la ira todavía no aplacada de Agamenón no les hubiese animado, ¿por qué razón iban a haber disparado aquellos cañones sobre las ruinas, en un páramo? Los nombres, no sólo un destino, son las cosas mismas. 'Absurdo y misterio en todo, Jacinta' dice el poeta José 
En esta ocasión, la paráfrasis supone una corrección del verso de Moreno Villa: allí donde el poeta ve misterio y absurdo ${ }^{12}$, el narrador cree que hay un misterio, pero "racional" y tejido sobre una trama de correspondencias y significados casi inefables, pero con la solidez que proporciona la identificación entre la palabra y la realidad con ella nombrada.

Volviendo a las obras de Vitaliano Brancati, éstas -además de su valor para ilustrar la personalidad de Majorana-también ayudan a transmitir al lector la atmósfera social que había en Italia cuando estaba el régimen fascista, época en la que tuvieron lugar los hechos narrados en el relato de investigación. Dos diálogos de la comedia Raffaele sirven para tal propósito. Al final del primer capítulo, tras la comunicación anónima que advierte a la policía de la sospecha de que la desaparición de Majorana esconde un complot contra los intereses nacionales, el narrador explica que es cierto que en aquella época los italianos fantaseaban sobre supuestos descubrimientos de Marconi que harían invencible a Italia en la guerra que se temía cercana: "E specialmente si favoleggiava di un "raggio della morte" che da Roma, per esperimento, era stato lanciato a fulminare una vaca situata a riceverlo in una radura nei pressi di Addis Abeba. Ne resta memoria in quella specie di "dizionario delle idee correnti" sotto il regime fascista che è la commedia Raffaele di Vitaliano Brancati" (Sciascia, La scomparsa 214) ${ }^{13}$.

A continuación, una conversación entre dos personajes de la obra de Brancati ilustra precisamente esa fantasía sobre el hipotético experimento por el cual un "rayo de la muerte" había matado una vaca en Etiopía. La definición de la comedia brancatiana como "diccionario de ideas corrientes"

Moreno Villa. En todo hay, sin embargo, misterio 'racional' de esencias y correspondencias, continua y tupida trama, de una cosa a otra, de un hombre a otro, de significados; apenas visibles, apenas decibles".

${ }^{12}$ Es el último verso del poema "Observaciones con Jacinta": "Mira, peliculera Jacinta, / mira bien lo que tiene por nariz el elefante. / Mira lo que necesitamos para sentarnos, / mira la casa inmensa que tiene lo que llamamos rey. / Mira esto de dormir, levantarse, dormir y / levantarse; / mira la mujer y el hombre que contratan no / separarse jamás; / mira al canalla, dueño de nuestro globo; / mira cómo la flor tierna sale del suelo duro; / mira que de los palos de los árboles / nacen comestibles aromáticos. / Mira que del cielo puro nos llegan / agua, rayo, luz, frío, calor, piedras, nieve. / Absurdo y misterio en todo, Jacinta" (Moreno Villa 100).

13 "Y se fantaseaba especialmente sobre un 'rayo de la muerte' que, como experimento, se habría lanzado desde Roma para fulminar una vaca situada para recibirlo en un descampado próximo a Addis Abebba. Queda constancia de ello en aquel 'diccionario de ideas corrientes' bajo el régimen fascista que es la comedia Raffaele de Vitaliano Brancati”. 
esconde además otra referencia literaria, pues con ella el narrador alude a la obra homónima de Gustave Flaubert (Dictionnaire des idées reçues), con la que ironizaba sobre los tópicos y clichés usados por la burguesía francesa del siglo XIX ${ }^{14}$. Con el ejemplo anterior del diálogo brancatiano, vemos que en lugar de acudir a fuentes historiográficas para retratar las preocupaciones y el sentir de la gente de la época, Sciascia ofrece la perspectiva de la literatura, con la convicción de que en la ficción puede reconocerse la verdad con mayor claridad que en una aproximación fidedigna de disciplinas como la historiografía.

Movido de nuevo por esta seguridad, y a propósito de las críticas de la prensa italiana a Fermi por no hacer el saludo fascista al recoger el premio Nobel en 1938, transcribe en una nota al pie otro diálogo cómico de Raffaele que ejemplifica los problemas que en aquella época podrían presentársele a quien, por error, estrechase la mano en lugar de hacer el obligado saludo romano (Sciascia, La scomparsa 218). También gracias a la citación intertextual se halla la respuesta a otros interrogantes relativos al caso investigado: ¿por qué la policía italiana fue incapaz de dar con el paradero de Majorana? ¿Qué elemento impedía que estuviera a la altura que exigía la investigación? Ofrece la solución "il discorso sul professor Cottard, sul medico, sui medici, che Bergotte fa nella Recherche" (Sciascia, La scomparsa 217) ${ }^{15}$, es decir, un personaje de En busca del tiempo perdido de Marcel Proust. El razonamiento de Bergotte es que las enfermedades de las personas inteligentes provienen, en gran medida, de su propia inteligencia y un médico como Cottard no podrá curarlas porque "ha previsto la dificultad de digerir las salsas, y las molestias gástricas, pero no ha previsto la lectura de Shakespeare (...) Le parecerá que tiene usted una dilatación de estómago

${ }^{14}$ Este tipo de intertextualidad, como ya indicamos, es empleada también para hablar de la voluntad de Majorana de vivir como "L'uomo solo", tomando la expresión del título de un cuento de Pirandello. Encontramos un tercer ejemplo en el fragmento donde se relata quiénes iban en el camarote en el trayecto entre Palermo y Nápoles, donde quizá el físico cedió su lugar a otra persona: "Siamo davvero al 'terzo uomo'. Ma il problema non è di difficile soluzione" (Sciascia, La scomparsa 256), es decir, "Nos encontramos ante el 'tercer hombre'. Pero el problema no es de difícil solución”. Había tres personas y falta por aclarar quién era Carlo Price y quién Ettore Majorana (o su sustituto) en esa confusión de identidades que se deduce del testimonio de Strazzeri; ante lo cual el narrador califica el asunto con el título de la novela The third man de Graham Greene.

15 "El razonamiento sobre el profesor Cottard, sobre el médico, sobre los médicos, que hace Bergotte en la Recherche". 
sin necesidad de reconocerle, porque ya la lleva en los ojos. Puede usted verla, se le refleja en los lentes" (Proust 167). Así lo vaticina Bergotte en la novela de Proust y el narrador lo proyecta en el caso de Majorana, donde la policía, al igual que Cottard, desde un principio ha etiquetado como falta de sentido común o locura las razones que explicarían la desaparición (Sciascia, La scomparsa 217).

La cita de la Recherche tiene un valor doble porque Bergotte nombra "la lectura de Shakespeare" como un factor imponderable para Cottard y, como sabemos por Edoardo Amaldi, el dramaturgo británico era junto con Pirandello el autor preferido por Majorana. De ahí que desde la lógica poética el narrador apele a sus obras -al igual que hace con Il fu Mattia Pascal o Uno, nessuno e centomila-y se apoye en ellas como elementos indispensables para un retrato completo de la figura del científico. Precisamente a continuación de aquella reflexión, aislada entre dos párrafos y en cursiva, la frase $I l$ resto è silenzio indica al lector que se encuentra ante otro ejemplo de intertextualidad:

possano avere avuto altro riflesso, negli occhiali di un commisario di polizia, negli occhiali dello stesso Bocchini, che quello del dissenno, della pazzia.

Il resto è silenzio.

Che Mussolini, informato e sollecitato da una "supplica" della madre di Ettore e da una lettera di Fermi, abbia chiesto a Bocchini il fascicolo dell'inchiesta... (Sciascia, La scomparsa 217) ${ }^{16}$.

Con este "Lo demás es silencio" la instancia narrativa parece indicar que, siendo el punto de partida una policía incapaz de comprender el drama de un hombre inteligente, es inevitable su fracaso en una investigación tan lastrada desde su inicio. Para expresar esa ineptitud, que condiciona cualquier otra consideración, reproduce las últimas palabras de Hamlet antes de morir ${ }^{17}$.

16 "[se nos hace imposible imaginar que el drama de un hombre inteligente, su voluntad de desaparecer, sus motivos] puedan haber tenido otro reflejo en las gafas de un comisario de policía, en las gafas del mismo Bocchini, que el de la falta de sentido común, el de la locura. El resto es silencio. Que Mussolini, informado e instado por una 'súplica' de la madre de Ettore y por una carta de Fermi hubiera pedido a Bocchini el expediente de la investigación..."

17 "No puedo vivir lo bastante para saber nuevas de Inglaterra, pero auguro que la elección recaerá en Fortinbrás; tiene a su favor mi voz moribunda. Díselo así con todos los incidentes, grandes y pequeños, que me han impulsado... ¡Lo demás es silencio!... ¡Oh!, ¡oh!, ¡oh!, ¡oh!... (mиеre)". (Shakespeare, Hamlet 288). 
En las páginas finales de La scomparsa di Majorana, la instancia narrativa vuelve a recurrir a la literatura shakesperiana. En el último capítulo-donde el narrador se convierte en un personaje y pasa a integrar de modo protagonista el relato de los hechos, alcanzando la identificación entre el tiempo del discurso y el tiempo del relato- la citación intertextual parece brotar más espontáneamente y ligada a la esfera de los recuerdos. El capítulo comienza con una cita tomada de La tempestad, que sufre una pequeña pero importante variación, pues el narrador la adecua a sus circunstancias:

La turpe cospirazione del bestiale Caliban contro la vita, mi è passata di mente. Una breve parola-mia, la mia vita-è volata via dalla battuta di Prospero: e così ce la ripetiamo andando dietro al padre certosino che guida la nostra visita a questo antico convento (...) La frase di Prospero batte nella memoria come tra nude pareti: La turpe cospirazione del bestiale Caliban contro la vita, mi è passata di mente. A momenti ne aggancia altre, dello stesso Prospero, nella stessa scena dell'atto IV de La tempesta, penultima opera di Shakespeare, ultima in un certo senso (Sciascia, La scomparsa 266) ${ }^{18}$.

En la memoria del narrador se repite continuamente la frase de Próspero ("¡Había olvidado la horrible conspiración del bruto de Calibán y de sus cómplices contra mi vida!” (Shakespeare, La tempestad 1011)), pero prescindiendo del determinante posesivo final ("contra la vida"). En la misma página del relato se añade otro extenso parlamento de este personaje, en el que termina afirmando que "estamos tejidos de idéntica tela que los sueños, y nuestra corta vida se halla envuelta por el sueño" (Shakespeare, La tempestad 1012). Una vez más, las palabras literarias desatan la argumentación del narrador, que parte de ellas y parafraseándolas intenta comprender la realidad, que parece adaptarse al molde creado por la literatura:

18 "La turbia conspiración del monstruo Caliban contra la vida me ha huido de la mente. Una breve palabra, $m i$, mi vida, se escapó de la parte del diálogo de Próspero; y así nos la repetimos mientras caminamos tras el padre cartujo que guía nuestra visita a este antiguo convento (...) La frase de Próspero golpea en la memoria como entre paredes desnudas: $L a$ turbia conspiración del monstruo Caliban contra la vida me ha huido de la mente. A ratos se une a otras, del mismo Próspero, de la misma escena del acto IV de La tempestad, penúltima obra de Shakespeare, última en un cierto sentido". 
Ma forse il richiamo dell'una battuta all'altra ha più a che fare col senso del nostro viaggio, della nostra visita: qualcuno qui, in questo convento, si è forse salvato dal tradire la vita tradendo la cospirazione contro la vita; ma la cospirazione non si è spenta per quella defezione, il dissolvimento continua, l'uomo sempre più si disgrega e svanisce in quella sostanza di cui sono fatti i sogni. E non è già un sogno di quel che l'uomo "era" l'ombra rimasta come stampata su qualche brandello di muro, a Hiroshima? (Sciascia, La scomparsa 267) ${ }^{19}$.

Por tanto, quizá Ettore Majorana se refugió en ese convento y así evitó participar en la conspiración contra la vida que eran las investigaciones atómicas, pero no por ello se detuvo esa conspiración. Al contrario y parafraseando al Próspero shakesperiano, el narrador añade que el hombre se disgrega cada vez más en la sustancia de la que están hechos los sueños. Prueba de ello sería la sombra de un hombre que quedó fijada en un muro de Hiroshima tras la explosión atómica.

Como podemos ver, unas ideas provocan otras y el narrador hace avanzar su discurso engarzándolas: las palabras del personaje de La tempestad son proyectadas en la vida de Majorana y de ésta se asciende a una consideración sobre el género humano. Es una observación de tintes apocalípticos, donde clama contra el proceso de barbarización que sufre el hombre, que se va convirtiendo en una sombra de sí mismo al atentar, como hizo en Hiroshima y Nagasaki, contra la realidad de su propia condición. Tras esta reflexión, el relato sugiere con la interrogación retórica una imagen que simboliza esa disolución -la sombra de un hombre fijada en una pared de la ciudad nipona,

19 "Aunque posiblemente ese paso de una frase del diálogo a la otra tiene más que ver con el sentido de nuestro viaje, de nuestra visita: quizá aquí, en este convento, alguien escapó de hacer traición a la vida traicionando la conspiración contra la vida; pero la conspiración no se extinguió por esa deserción, la disolución sigue adelante, el hombre se disgrega cada vez más en la misma sustancia de la que están hechos los sueños. Y ¿acaso no es ya un sueño de lo que era el hombre, esa sombra que quedó como estampada en un trozo de pared, en Hiroshima?" 
después del gran estallido ${ }^{20}$ - y en la que es posible hallar resonancias poéticas de Giuseppe Ungaretti y de Eugenio Montale ${ }^{21}$.

Por esto último, unido a la trascendencia semántica que adquieren en la configuración del propio texto las citas de Eliot, Proust, Pirandello o Brancati, cabría inferir, como señala Francesca Vennarucci, que por medio de la citación intertextual 'Sciascia pone en relación la 'disgregación' de Majorana con la compleja 'disgregación' del sujeto que atraviesa y caracteriza gran parte de la literatura del siglo XX: el drama interior del joven físico se convierte en emblema de la desazón existencial y filosófica del hombre del siglo $\mathrm{XX}$, privado de certezas y de referencias en un realidad cambiante y engañosa" (141).

\section{DIGRESIONES DE CRÍTICA LITERARIA}

Aunque es un recurso tangencial y externo -pues no implica la inclusión de fragmentos de otras obras literarias en el relato de La scomparsa di Majorana-podemos considerar como una forma menor de intertextualidad

${ }^{20}$ Esta imagen se asemeja a una idea expresada por Albert Camus y que Sciascia suscribía, esgrimiéndola como razón por la que había escrito La scomparsa di Majorana: "Vivir pegado a la pared es llevar una vida de perros. Pues bien, los hombres de mi generación y los de la generación que hoy entra en la facultad y los talleres han vivido como perros y viven cada vez más como perros. Gracias a la ciencia, sobre todo gracias a la ciencia" (Cit. en Ritter Santini 73).

${ }^{21}$ En "Vanità" (incluido en Naufragi 1919), Ungaretti escribe: "D'improvviso / è alto / sulle macerie / il limpido / stupore / dell'immensità // E l'uomo / curvato / sull'acqua / sorpresa / dal sole / si rinviene / un'ombra // Cullata e / piano / franta" (Ungaretti 107-108). ("De improviso / está, alto, / sobre las ruinas / el límpido / estupor / de la inmensidad // Y el hombre / encorvado / sobre el agua / sorprendida / por el sol / se descubre / una sombra // Mecida y / despacio / rota"). La imagen de la sombra y el muro también está presente en uno de los poemas de Ossi di sepia de Montale. En concreto, en el mismo del que ya hemos reproducido la última estrofa (cfr. supra nota 9), y en cuyas dos anteriores dice: "Non chiederci la parola che squadri da ogni lato / l'animo nostro informe, e a lettere di fuoco lo dichiari e risplenda come un croco / perduto in mezzo a un polveroso prato. // Ah l'uomo che se ne va sicuro, / agli altri ed a se stesso amico, / e l'ombra sua non cura che la canicola / stampa sopra uno scalcinato muro!" (Montale 29) ("No nos pidan la palabra que de cada lado / escudriñe nuestro ánimo informe, y con letras de fuego lo declare y lo haga resplandecer como un azafrán/perdido en medio de un prado polvoroso // ¡ Ah, el hombre que se va seguro, / a los otros y así mismo amigo, / y su sombra no cuida más que la canícula/ fija sobre un desconchado muro!"). 
las divagaciones que realiza la instancia narrativa sobre acontecimientos y anécdotas del ámbito literario o en torno a la figura y obra de algunos escritores. Es un recurso empleado para contextualizar o establecer una relación de analogía con aquello que está narrando o bien por el deleite y el valor concedido al mismo acto de la digresión.

En el capítulo sexto del racconto-inchiesta, por ejemplo, el narrador traza un panorama general de la Italia de los años treinta para reflejar cómo el entusiasmo por el régimen fascista imperaba en todas las facetas de la vida pública nacional (Sciascia, La scomparsa 243). Aunque no tenga relación directa con la vida de Majorana ni con su investigación, la atención reservada a la literatura es mucho mayor que la dedicada a otras manifestaciones culturales y sociales. En el esbozo de los rasgos principales del círculo literario de la época, la instancia narrativa se refiere con ambigüedad-excepto con Gabrielle D'Annunzio- a los escritores simpatizantes con el régimen de Mussolini, como si evitara nombrarlos para que no formen parte de la constelación de referencias literarias de La scomparsa di Majorana, un derecho reservado a los autores admirados por Sciascia. Éstos, en cambio, transitan por el relato y, ya sea en un orden temático o estilístico, dejan una huella apreciable. Incluso sus circunstancias vitales pueden ayudar al narrador a adentrarse en el carácter del físico siciliano desaparecido. De este modo, una explicación de la trayectoria vital de Stendhal y su intento de postergar la hora de escribir es la clave para comprender la personalidad de Majorana y su análoga voluntad de retrasar el cumplimiento de sus investigaciones:

Prendiamo Stendhal. È un caso, il suo, di precocità ritardata al possibile (...) Perde tempo. Si finge ambizioni carrieristiche e mondane. Si nasconde. Si maschera. Rampa per plagi e pseudonimi (che sono poi il rovescio e il dritto della stessa paura). Ed è un gioco che fino ad un certo punto gli riesce (...) Senza saperlo, senza averne coscienza, come Stendhal Majorana tenta di non fare quel che deve fare, quel che non può non fare (Sciascia, La scomparsa 225-227) ${ }^{22}$.

22 "Tomemos a Stendhal. El suyo es un caso de precocidad retardada en lo posible. Pierde tiempo. Finge tener ambiciones de hacer carrera y también mundanas. Se esconde. Se enmascara. Va dando tumbos entre plagios y pseudónimos (que no son más que el anverso y el reverso del mismo temor). Y es un juego que, hasta cierto punto, le sale bien (...) Sin saberlo, sin ser consciente de ello, Majorana, como Stendhal, intenta no hacer lo que debe hacer, lo que no puede dejar de hacer". 
La comparación entre el escritor y el científico no solo ilumina la vida de este último, sino que se convierte paralelamente en un ejercicio de crítica literaria, en el cual el narrador atribuye a esa precocidad aplazada hasta la madurez el encanto de las páginas stendhalianas (Sciascia, La scomparsa 225). En ese mismo capítulo, después de transcribir la detallada descripción de Edoardo Amaldi sobre Majorana, para completar su retrato y el lector pueda imaginarse la fisonomía del joven, el narrador recurre al "orden de las semejanzas"23, invocando el nombre de Giuseppe Antonio Borgese, un escritor con quien el científico guardaba parecido físico (Sciascia, La scomparsa 222).

En definitiva, todo lo relativo a la literatura, en sus más diversas manifestaciones, es susceptible de ser integrado en el relato por sus posibilidades como instrumento epistemológico o porque emerge en la memoria de la instancia narrativa y permite hacer avanzar el relato. Así, el análisis de un documento policial sobre la desaparición de Majorana en el que hay anotaciones con tintas de diversos colores provoca el recuerdo de los hábitos de los autores decimonónicos, pues algunos escribían en tinta violeta (Sciascia, La scomparsa 212). O bien, la paráfrasis de una idea de Alberto Savinio sirve para elogiar y reivindicar a pie de página la obra de este autor, al que califica como "il più grande scrittore italiano tra le due guerre" y que, sin embargo, cada vez es menos conocido en su país. Según la opinión del narrador, se debe a que entre sus lectores no tienen cabida los mediocres o imbéciles y el número de éstos va en aumento en Italia. De ahí su esperanza de que al menos en Francia pueda ser leído como merece (Sciascia, La scomparsa 269).

Tras nombrar al autor de la Nuova enciclopedia y a propósito del verso "Assurdo e mistero in tutto, Giacinta" del poeta español Moreno Villa, el relato del racconto-inchiesta se entrega a la divagación en otra nota al pie, que nos parece conveniente reproducir aquí por completo, para apreciar el tono y los giros que toma la narración:

23 Así tituló Sciascia, "L’ordine delle somiglianze", un ensayo incluido en el libro Cruciverba (1983) en el que reflexionaba sobre la obra pictórica de Antonello de Messina. Retomando una consideración del escritor Antonio Castelli acerca del "orden bioétnico de semejanzas", Sciascia concluía que el juego de los parecidos es un instrumento de conocimiento (Sciascia, Cruciverba 989-990) y que la vida y la realidad serían incognoscibles si el arte, a través de sus arquetipos de verdad, como en el caso de los retratos de Antonello, no estableciese con ello correspondencias y conexiones, ordenando los acontecimientos dentro de una racional jerarquía de semejanzas, dentro de un sistema teleológico. 
Tanto per continuare al modo di Savinio: questo verso, che resta indelebile nella memoria, grazie a quel nome femminile da noi poco consueto anche se Capuana ne fece il titolo di un romanzo niente male (José Moreno Villa dà a Jacinta l'attributo di "peliculera" -parola intraducibile se non con le espressioni patita del cinema, invasata del cinema e dei suoi miti, aspirante a far del cinema; ma che Montale, per esigenza di verso, traduce in "fotogenica"); questo verso potrebbe riassumere tutta la poesia di Moreno Villa, se si facesse quel gioco cretino che tra futurismo e frammentismo qualcuno ha fatto sulla poesia italiana: un verso che sia tutto un poeta, un verso da salvare in una microscopica antologia. E fu fatta eccezione per il solo Dante, di cui se ne salvarono due. Questi giochi cretini è però sintomatico che vengano proposti nei momenti disperati: come in questo dopoguerra, quando venne fuori quello dei dieci libri da salvare -da salvare dalla distruzione atomica. Come se bastasse salvare i dieci libri, se poi non si salvano gli uomini in grado di leggerli. E così, questo breve giro alla Savinio, ci ha riportati al nostro tema (Sciascia, $L a$ scomparsa 269) ${ }^{24}$.

El nombre de Savinio aparece al principio y al final de la nota, bajo su influencia se abre y se cierra este fragmento marcado por la divagación, donde el relato avanza a través de las asociaciones mentales entre motivos diversos: el nombre de Giacinta recuerda el título homónimo de una novela de Luigi Capuana, la cualidad de "peliculera" que caracteriza a aquélla origina una disquisición sobre el problema de traducir esta palabra al italiano y la opción que escogió Eugenio Montale... Vuelve al verso de Moreno Villa,

24 "Para continuar a la manera de Savinio, he aquí este verso que se nos quedó indeleble en la memoria gracias a ese nombre de mujer tan poco corriente entre nosotros, aunque Capuana hiciera de él el título de una novela bastante buena, José Moreno Villa atribuyó a Jacinta la cualidad de 'peliculera', palabra intraducible al italiano si no es con la expresión 'loca por el cine', obsesionada por el cine y sus mitos, aspirante a hacer cine; pero que Montale, por exigencia de la rima, traduce como 'fotogénica'; este verso podría resumir toda la poesía de Moreno Villa si se llevara a cabo ese juego cretino que, entre futurismo y fragmentismo, ha hecho alguno sobre la poesía italiana: un verso que sea todo un poeta, un verso para salvar en una antología microscópica. Y se hizo una excepción únicamente con Dante, del cual se salvaron dos. Es, sin embargo, sintomático que estos juegos cretinos sean propuestos en momentos desesperados, como en esta posguerra, cuando surge aquello de los diez libros que salvar, que salvar de la destrucción atómica. Como si sirviera de algo salvar los diez libros, si luego no se salvan los hombres en condiciones de leerlos. Y así, este breve circunloquio a la manera de Savinio, nos ha devuelto a nuestro tema". 
que según el narrador podría valer como resumen de toda su obra, pero eso sería caer en un juego cretino (un verso que sea todo un poeta, un verso para salvar en una antología microscópica), de esos que siempre surgen en momentos desesperados, como la posguerra. De nada sirve salvar los libros, si los hombres que podrían leerlos son víctimas de la destrucción atómica. Así, el relato vuelve al tema que le ocupa, después de hacer questo breve giro alla Savinio.

El giro saviniano recorre varios referentes literarios y al mismo tiempo reflexiona sobre el valor cívico de la literatura y los reduccionismos a los que se ve sometida cuando la civilización de la que es expresión se tambalea. Por otra parte, el fragmento demuestra hasta qué punto Sciascia ha convertido en un rasgo estilístico propio aquellas peculiaridades que él estimaba en Savinio y a las que se refería así:

Sapeva conversare col "compagno leggero", con "l'amico stendhaliano", col lettore leggero e stendhaliano (era sicuro dei suoi lettori, dell'intelligenza, della leggerezza e dello stendhalismo dei suoi lettori; ma è da intendere lo stendhalismo, in Savinio, come sinonimo di dilettantismo, del saper dilettarsi specialmente di quelle cose di cui i più non si dilettano). La sua conversazione era insomma un modo della libertà: un rompere le regole, le convenzioni, i compromessi, le "idee ricevute", le categorie, i generi (Sciascia, Alla cinque 165$166)^{25}$.

Como queda patente en los ejemplos anteriores, con la predilección por las digresiones de contenido literario y apelando a la inteligencia de sus lectores, también Sciascia trasciende las convenciones y limitaciones del género. Su racconto-inchiesta no se reduce a la investigación de la vida y desaparición de Ettore Majorana según los parámetros historiográficos al uso, sino que articula su indagación sobre referentes literarios que, pese a su disparidad y aparente gratuidad en ocasiones, son el fundamento y la clave de su acercamiento a la verdad.

25 'Sabía conversar con el 'compañero ligero', con el lector ligero y stendhaliano (estaba convencido de la inteligencia, ligereza y stendhalismo de sus lectores; pero hay que entender el stendhalismo, en Savinio, como sinónimo de diletantismo, del saber deleitarse, especialmente de aquellas cosas en las que la mayoría no encuentra deleite). Su conversación era, en definitiva, un modo de libertad: un romper las reglas, las convenciones, los compromisos, las 'ideas corrientes', las categorías, los géneros". 


\section{CONCLUSIONES}

En La scomparsa di Majorana el acontecimiento real de la misteriosa desaparición en 1938 del científico italiano Ettore Majorana es el punto de partida y motivo central de la investigación emprendida por el narrador, que emplea en su reconstrucción un amplio repertorio de fuentes historiográficas y documentos verídicos, leídos e interpretados con una mirada filológica. Ante la insuficiencia de éstos, apela a un plus de sentido que solo puede provenir de una perspectiva eminentemente literaria y que se materializa a través de la intertextualidad con obras de ficción. No son un juego gratuito de referencias invocadas solo por el placer de la citación o la erudición, sino instrumentos en la busca de una verdad que halla en la narración un cumplimiento que la realidad contingente no puede ofrecer.

Partiendo del precepto defendido por Dupin (el investigador nacido de la pluma de E. A. Poe según el cual era indispensable en toda indagación la capacidad de identificarse con el otro), y sabiendo que los autores predilectos de Ettore Majorana eran Pirandello y Shakespeare, Sciascia intenta, a través de las obras de éstos, adentrarse en el carácter misántropo del físico siciliano. Así pues, personajes pirandellianos como Mattia Pascal y Vitangelo Moscarda se erigen como posibles modelos del plan de desaparición que habría diseñado Majorana; una frase de Hamlet o un fragmento de La tempestad asumen la voz del narrador en determinados pasajes de su inchiesta. De igual modo, extendiendo el repertorio de fuentes poéticas con que iluminar la realidad, un diálogo extraído de la comedia Raffaele de Vitaliano Brancati ilustra la vida cotidiana en la Italia fascista, una descripción proustiana tomada de En busca del tiempo perdido sirve como metáfora de la actitud policial, el drama personal de Ettore Majorana puede sintetizarse en la paráfrasis de un verso de T. S. Eliot.

Esta perspectiva literaria también informa la narración y la estructura de la obra. Se manifiesta en el plano discursivo, articulado como un relato policíaco en donde el narrador-detective ha de enfrentarse a la resolución de un misterio, la muerte o huida del joven físico, por medio de una lectura clarividente de los documentos relativos al caso y con la ayuda de la rememoración literaria. Sciascia pretende restituir el verdadero sentido de los hechos, lo cual no necesariamente implica que éstos sean presentados con claridad; y por eso el resultado fáctico puede parecer un fracaso. Sin embargo, aunque sea misteriosa o siga velada por la oscuridad, el escritor cree haber hallado la verdad. Por su parte, la caracterización de Majorana 
se aproxima a la de un personaje ficticio, pero sin asumir definitivamente esa condición. La ficcionalización de la realidad nunca se completa, de tal modo que el relato se desarrolla en un espacio que fluctúa ambiguamente entre lo histórico y lo ficticio, alcanzando un difícil equilibrio en el cual, sin confundirse uno y otro, nutren una obra que no es ni una mera crónica de hechos verídicos ni una novela histórica. Es un equilibrio difícil que Sciascia sabe sostener y en el que radica el valor de su propuesta creativa, pues "si La scomparsa di Majorana fuese una obra totalmente "histórica" sería poco convincente; si fuese una obra totalmente "de invención", tendría corto aliento" (Traina 198).

Por todo ello, La scomparsa di Majorana adquiere una relevancia singular dentro del corpus sciasciano, en un doble sentido. Por un lado, sus propios méritos literarios lo convierten en un racconto-inchiesta emblemático de la manera en que el autor cultivó el género, mostrando las posibilidades formales y epistemológicas que podía alcanzar con un relato de investigación. Por otro, supone un hito incomparable dentro de su concepción acerca del valor de la literatura, pues es entonces cuando Sciascia toma conciencia de que ésta es la forma más plena en que la verdad puede encarnarse.

\section{BIBLIOGRAFÍA}

Alighieri, Dante. Divina Commedia, 1472. Firenze: La Nuova Italia Editrice, 1976.

Del Prado Biezma, Javier. "Francia en Fortunata y Jacinta". Imágenes de Francia en las letras hispánicas. Ed. Francisco Lafarga. Barcelona: PPU, 1989. 405-416.

Eliot, Thomas Stearns. La tierra baldía. 1922. Madrid: Editorial Swan, 1985.

Magueijo, Joao. A Brilliant Darkness: The Extraordinary Life and Mysterious Disappearance of Ettore Majorana, the Troubled Genius of the Nuclear Age. New York: Basic Books, 2009.

Manzoni, Alessandro. Historia de la columna infame. 1842. Barcelona: Bruguera, 1984.

Millán Alba, José Antonio. "En torno al concepto de intertextualidad". Europa en España, España en Europa: actas del Simposio Internacional de Literatura Comparada. Coord. Hugo Dysenrick et al. Barcelona: PPU, 1990. 11-20.

Montale, Eugenio. Tutte le poesie. Verona: Arnoldo Mondadori, 2000.

Moreno Villa, Jacinta, Jacinta la pelirroja. 1929. Madrid: Castalia, 2000.

Nigro, Salvatore Silvano, comp. Leonardo Sciascia scrittore editore, Palermo: Sellerio, 2003.

Pirandello, Luigi. "Uno, nessuno e centomila". Opere. Vol. "Tutti i romanzi", Verona: Mondadori, 1966. 1283-1416. 
Ficción, historia y verdad: estudio de la intertextualidad en La scomparsa...

Proust, Marcel. En busca del tiempo perdido. 2, A la sombra de las muchachas en flor. 1919. Madrid: Alianza, 1966.

Recami, Erasmo. Il caso Majorana. Epistolario, documenti, testimonianze. Roma: Di Renzo, 2000.

Russo, Bruno. Ettore Majorana. Un giorno di marzo. Palermo: Flaccovio, 1997.

Ritter Santini, Lea. "Un desgarrón en el cielo de papel”. Ensayo incluido en Sciascia, Leonardo. La desaparición de Majorana, Barcelona: Juventud, 1994. 71-92.

Sciascia, Leonardo. La Sicilia come metafora. Milano: Mondadori, 1979.

"La scomparsa di Majorana". 1975. Opere 1971-1983. Ed. Claude Ambroise, Milano: Bompiani, 1989. 205-270.

"L'affaire Moro". 1978. Opere 1971-1983. Ed. Claude Ambroise. Milano: Bompiani, 1989. 463-565.

“Cruciverba”. 1983. 1971-1983. Ed. Claude Ambroise. Milano: Bompiani, 1989. $965-1282$

“Alla cinque da Savinio". L'adorabile Stendhal. Ed. Maria Andronico. Milano: Adelphi, 2003. 165-170.

Shakespeare, William. "Hamlet". Obras completas. Tomo II. Madrid: Aguilar, 1974. 219289.

“La tempestad". Obras completas. Tomo II. Madrid: Aguilar, 1974. 979-1023.

Traina, Giuseppe. Sciascia. Bruno Mondadori: Milano, 1999.

Ungaretti, Giuseppe. Vita d'un uomo: poesie. Vol. "L'allegria". Verona: Arnoldo Mondadori, 1970.

Vecellio, Valter, comp. L'uomo solo. L'affaire Moro di Leonardo Sciascia, "Quaderni Leonardo Sciascia”, n 7, Milano: La Vita Felice, 2002.

Vennarucci, Francesca. "Ettore Majorana e il mito del rifiuto della scienza". L'enciclopedia di Leonardo Sciascia. Caos, ordine, caso. Ed. Pietro Milone. "Quaderni Leonardo Sciascia", $\mathrm{n}^{\mathrm{o}}$ 11, Milano: La Vita Felice, 2007. 131-152. 